九州大学学術情報リポジトリ

Kyushu University Institutional Repository

\title{
Natural Habitats and Geographic Distribution of Diploid Lilium lancifolium in Islands of the Bay of Kyunggi, Korea
}

Kim, Jong Hwa

Kangwon National University

Xuan, Yong Hao

Kangwon National University

Hiramatsu, Michikazu

Faculty of Agriculture, Kyushu Unviersity

Okubo, Hiroshi

Faculty of Agriculture, Kyushu Unviersity

https://doi.org/10.5109/4672

出版情報：九州大学大学院農学研究院紀要. 50 (2)，pp.593-600，2005-10-01. Faculty of Agriculture, Kyushu University

バージョン：

権利関係： 


\title{
Natural Habitats and Geographic Distribution of Diploid Lilium lancifolium in Islands of the Bay of Kyunggi, Korea
}

\author{
Jong Hwa KIM ${ }^{1 *}$, Yong Hao XUAN ${ }^{1}$, Michikazu HIRAMATSU \\ and Hiroshi OKUBO \\ Laboratory of Horticultural Science, Division of Agricultural Botany, \\ Department of Plant Resources, Faculty of Agriculture, \\ Kyushu University, Fukuoka 812-8581, Japan \\ (Received June 30, 2005 and accepted July 26, 2005)
}

\begin{abstract}
Habitats and geographic distribution were investigated for diploid and triploid $L$. lancifolium grown in 16 islands of the Bay of Kyunggi, the western region of South Korea. Most of natural populations were found at the coastal areas in the islands: $103(72.5 \%)$ of 142 natural populations inhabited in sea cliffs, 28 (18.4\%) in beaches, and the remainder in forests, roadside, riverside, grassy slopes and gardens. Among 16 islands investigated, 13 islands located in the southern and central area of the bay were inhabited by diploid populations alone. Kanghwa-do, the nearest island to the mainland of South Korea, was inhabited by eight diploid and six triploid populations. Backryung-do and Sochung-do, the northernmost islands in South Korea, were inhabited by triploid populations alone. Combined with previously demonstrated facts on ploidy distribution of $L$. lancifolium, the results suggest that diploid $L$. lancifolium is indigenous to the middle western to southern islands and coasts of the Korean Peninsula, and Kanghwa-do and the neighbor islands are approximate northern limit of the distribution of diploid L. lancifolium.
\end{abstract}

\section{INTRODUCTION}

It has been well confirmed that tiger lily, Lilium lancifolium (synonym $L$. tigrinum), is a polyploid complex involving both diploid and triploid forms (Noda, 1978, $1986,1991)$. Noda (1986) hypothesized from his cytological studies that the triploid form may be either the allotriploid produced by natural hybridization between the diploid form and closely related diploid species such as L. leichtlinii var. maximowiczii, or the autotriploid directly originated from the diploid form through the production of unreduced gametes.

To understand the origin of triploid in nature, entire natural distribution of diploid and triploid forms must be investigated. Several reports (Willson, 1925; Lightly, 1968; Noda, 1986; Noda and Lee, 1980; Song, 1997) mentioned the distribution of $L$. lancifolium in Korea, but, little has been known about exact geographic distribution of each ploidy form. Noda (1991) reported that the diploid form is confined to the southern part of Korea including two relatively large islands, Cheju Island (South Korea) and Tsushima Islands (Japan), whereas the triploid form is widely distributed in East Asia. We previously found the fact that the reproductive individuals of L. lancifolium

\footnotetext{
1 Department of Horticultural Science, Kangwon National University, Chuncheon 200-701, Korea

* Corresponding author (E-mail: jonghwa@kangwon.ac.kr)
} 
frequently set capsules in western islands and coasts of South Korea, indicating that diploid forms are common in those regions (Kim et al., 2004).

This study demonstrates the natural habitats and geographic distribution of diploid $L$. lancifolium in islands of the Bay of Kyunggi located in the western region of South Korea.

\section{MATERIALS AND METHODS}

Populations of $L$. lancifolium were investigated in 16 islands of the Bay of Gyunggi located in the western region of the Korean Peninsula from 2000 to 2003 . Of all the islands, autogenous populations of $L$. lancifolium were found in the coastal areas but not in the inland. Approximate number of reproductive individuals and frequency of capsule set were investigated for each population. The populations geographically isolated at least more than $100 \mathrm{~m}$ distance from other populations were judged as independent populations. The ploidy level was determined by the combination of counting chromosome number of root tip cells and measuring relative DNA content of leaf cells with flow cytometer (Partec PA Ploidy Analyzer, Germany).

Somatic chromosomes in root-tips were observed by the aceto-carmine squash method as follows: Root tips of the two representative plants from each island were pretreated with $0.002 \mathrm{M} 8$-hydroxyquinolin at room temperature for $24 \mathrm{hrs}$, fixed with ethanol acetic acid (3:1) at $5^{\circ} \mathrm{C}$ for $1 \mathrm{hr}$, hydrolyzed with $1 \mathrm{~N} \mathrm{HCl}$ at $60^{\circ} \mathrm{C}$ for $5 \mathrm{~min}$. The macerated root tips were placed on a glass slide with a few drops of $1 \%$ carmine solution diluted with $45 \%$ acetic acid at room temperature for 5 to $15 \mathrm{~min}$, squashed under a cover glass, then observed with a microscope. Plants cytologically estimated to be diploid and triploid were used as controls for flow cytometric analysis.

Procedure of flow cytometric analysis followed that by Ozaki et al. (1998). Leaf tissues of three plants collected from each population were chopped with a sharp razor blade in a plastic Petri dish with $500 \mu \mathrm{L}$ nucleus-isolation buffer (High resolution DNA kit, Partec), and the suspension was filtered through a $30 \mu \mathrm{m}$ mesh filter. Then, $1 \mathrm{~mL}$ staining solution (High resolution DNA kit, Partec) containing 4'-6-diamidiono-2-phenylindole (DAPI) was added into the filtered solution. The prominent signal peak for diploid and triploid was adjusted at channel 100 and 150 , respectively.

\section{RESULTS AND DISCUSSION}

\section{Habitat and putative dispersion mode of insular $L$. lancifolium}

We found 142 populations in 16 islands of the Bay of Kyunggi. The localities and descriptions of the natural habitat, and the ploidy form for each population are summarized in Table 1. Population sizes varied considerably with 10 to 1,000 reproductive individuals per population. Relatively large and well-reserved natural populations were found in the coast of Acha-do (Isl. No. 3) and Ahnmyun-do (Isl. No. 16).

Among $142 \mathrm{~L}$. lancifolium populations, $103(72.5 \%)$ were growing on cliffs by the sea and 28 (19.7\%) were on beaches (Table 2). Distribution in forests, at roadside, riverside, gardens and grassy slopes occupied below $10 \%$ in these islands, although such habitats are common in the inland of South Korea (Kim et al., 2004). These growth 
Table 1. Localities, habitats, and ploidy forms of $L$. lancifolium populations found in 16 islands in the Bay of Kyunggi.

\begin{tabular}{|c|c|c|c|c|c|}
\hline $\begin{array}{l}\text { Island } \\
\text { No. }\end{array}$ & Population & $\begin{array}{l}\text { County, } \\
\text { province }\end{array}$ & $\begin{array}{l}\text { Island, locality } \\
\text { and habitat }\end{array}$ & $\begin{array}{l}\text { Population } \\
\text { size }^{y}\end{array}$ & $\begin{array}{l}\text { Ploidy } \\
\text { form }\end{array}$ \\
\hline \multirow[t]{10}{*}{1} & A & Incheon, Ongjin-gun & Backryung-do, Jincheonri, Sea cliff & 100 & Triploid \\
\hline & $\mathrm{B}$ & Incheon, Ongjin-gun & Backryung-do, Jincheonri, Sea cliff & 100 & Triploid \\
\hline & $\mathrm{C}$ & Incheon, Ongjin-gun & Backryung-do, Sahangpo, Sea cliff & 50 & Triploid \\
\hline & $\mathrm{D}$ & Incheon, Ongjin-gun & Backryung-do, Dumoojin, Sea cliff & 50 & Triploid \\
\hline & $\mathrm{E}$ & Incheon, Ongjin-gun & Backryung-do, Yeonhwari, Sea cliff & 50 & Triploid \\
\hline & $\mathrm{F}$ & Incheon, Ongjin-gun & Backryung-do, Joonghwadong, Riverside & 50 & Triploid \\
\hline & G & Incheon, Ongjin-gun & Backryung-do, Bookpori, Seaside & 50 & Triploid \\
\hline & $\mathrm{H}$ & Incheon, Ongjin-gun & Backryung-do, Mt. Yonggiwon, Seaside & 50 & Triploid \\
\hline & I & Incheon, Ongjin-gun & Backryung-do, Mt. Yonggiwon, Seaside & 50 & Triploid \\
\hline & $\mathbf{J}$ & Incheon, Ongjin-gun & Backryung-do, Kongdol beach, Seaside & 50 & Triploid \\
\hline \multirow[t]{10}{*}{2} & A & Incheon, Ongjin-gun & Sochung-do, Yaedong, Sea cliff & 50 & Triploid \\
\hline & $\mathrm{B}$ & Incheon, Ongjin-gun & Sochung-do, Nohwadong, Sea cliff & 50 & Triploid \\
\hline & $\mathrm{C}$ & Incheon, Ongjin-gun & Sochung-do, Tapdong, Sea cliff & 50 & Triploid \\
\hline & $\mathrm{D}$ & Incheon, Ongjin-gun & Sochung-do, Tapdong, Sea cliff & 50 & Triploid \\
\hline & $\mathrm{E}$ & Incheon, Ongjin-gun & Sochung-do, Boonampogu, Sea cliff & 50 & Triploid \\
\hline & $\mathrm{F}$ & Incheon, Ongjin-gun & Sochung-do, Boonampogu, Sea cliff & 50 & Triploid \\
\hline & $\mathrm{G}$ & Incheon, Ongjin-gun & Sochung-do, Boonampogu, Sea cliff & 50 & Triploid \\
\hline & $\mathrm{H}$ & Incheon, Ongjin-gun & Sochung-do, Ajinpogu, Sea cliff & 50 & Triploid \\
\hline & I & Incheon, Ongjin-gun & Sochung-do, Ajinpogu, Sea cliff & 50 & Triploid \\
\hline & $\mathrm{J}$ & Incheon, Ongjin-gun & Sochung-do, Ajinpogu, Sea cliff & 50 & Triploid \\
\hline \multirow[t]{19}{*}{3} & $\mathrm{~A}$ & Incheon, Kanghwa-gun & Acha-do, Sea cliff & 200 & Diploid \\
\hline & $\mathrm{B}$ & Incheon, Kanghwa-gun & Acha-do, Sea cliff & 200 & Diploid \\
\hline & $\mathrm{C}$ & Incheon, Kanghwa-gun & Acha-do, Sea cliff & 200 & Diploid \\
\hline & $\mathrm{D}$ & Incheon, Kanghwa-gun & Acha-do, Sea cliff & 200 & Diploid \\
\hline & $\mathrm{E}$ & Incheon, Kanghwa-gun & Acha-do, Sea cliff & 200 & Diploid \\
\hline & $\mathbf{F}$ & Incheon, Kanghwa-gun & Acha-do, Sea cliff & 200 & Diploid \\
\hline & G & Incheon, Kanghwa-gun & Acha-do, Sea cliff & 200 & Diploid \\
\hline & $\mathrm{H}$ & Incheon, Kanghwa-gun & Acha-do, Sea cliff & 100 & Diploid \\
\hline & I & Incheon, Kanghwa-gun & Acha-do, Sea cliff & 100 & Diploid \\
\hline & $\mathrm{J}$ & Incheon, Kanghwa-gun & Acha-do, Sea cliff & 100 & Diploid \\
\hline & $\mathrm{K}$ & Incheon, Kanghwa-gun & Acha-do, Sea cliff & 100 & Diploid \\
\hline & $\mathrm{L}-\mathrm{I}$ & Incheon, Kanghwa-gun & Acha-do, Sea cliff & 100 & Diploid \\
\hline & $\mathrm{L}-\mathrm{II}$ & Incheon, Kanghwa-gun & Acha-do, Sea cliff & 100 & Diploid \\
\hline & M & Incheon, Kanghwa-gun & Acha-do, Sea cliff & 100 & Diploid \\
\hline & $\mathrm{N}$ & Incheon, Kanghwa-gun & Acha-do, Sea cliff & 300 & Diploid \\
\hline & 0 & Incheon, Kanghwa-gun & Acha-do, Sea cliff & 300 & Diploid \\
\hline & $\mathrm{P}$ & Incheon, Kanghwa-gun & Acha-do, Sea cliff & 300 & Diploid \\
\hline & Q & Incheon, Kanghwa-gun & Acha-do, Sea cliff & 300 & Diploid \\
\hline & $\mathrm{R}$ & Incheon, Kanghwa-gun & Acha-do, Sea cliff & 100 & Diploid \\
\hline \multirow[t]{5}{*}{4} & A & Incheon, Kanghwa-gun & Suckmo-do, Josanggot, Sea cliff & 10 & Diploid \\
\hline & $\mathrm{B}$ & Incheon, Kanghwa-gun & Suckmo-do, Josanggot, Sea cliff & 10 & Diploid \\
\hline & $\mathrm{C}$ & Incheon, Kanghwa-gun & Suckmo-do, Eoreujung, Sea cliff & 10 & Diploid \\
\hline & $\mathrm{D}$ & Incheon, Kanghwa-gun & Suckmo-do, Eoreujung, Sea cliff & 50 & Diploid \\
\hline & $\mathrm{E}$ & Incheon, Kanghwa-gun & Suckmo-do, Eoreujung, Sea cliff & 20 & Diploid \\
\hline \multirow[t]{5}{*}{5} & A & Incheon, Kanghwa-gun & Kanghwa-do, Gilsang, Donggumri, Sea cliff & 30 & Diploid \\
\hline & $B-I$ & Incheon, Kanghwa-gun & Kanghwa-do, Hwado, Dongmakri, Grassy slope & 20 & Diploid \\
\hline & $\mathrm{B}-\mathrm{II}$ & Incheon, Kanghwa-gun & Kanghwa-do, Hwado, Dongmaksunsudae, Beach & 20 & Diploid \\
\hline & $\mathrm{C}$ & Incheon, Kanghwa-gun & Kanghwa-do, Hwado, Naeri, Grassy slope & 20 & Diploid \\
\hline & $\mathrm{D}$ & Incheon, Kanghwa-gun & Kanghwa-do, Yangdo, Gunpyungri, Roadside & 15 & Diploid \\
\hline
\end{tabular}


Table 1. Continued.

\begin{tabular}{|c|c|c|c|c|c|}
\hline $\begin{array}{l}\text { Island } \\
\text { No. }^{z}\end{array}$ & Population & $\begin{array}{l}\text { County, } \\
\text { province }\end{array}$ & $\begin{array}{l}\text { Island, locality } \\
\text { and habitat }\end{array}$ & $\begin{array}{l}\text { pulation } \\
\text { size }^{y}\end{array}$ & $\begin{array}{c}\text { Ploidy } \\
\text { form }\end{array}$ \\
\hline \multirow{19}{*}{6} & $\mathrm{E}$ & Incheon, Kanghwa-gun & \multicolumn{2}{|l|}{ Kanghwa-do, Yangdo, Hwangkol, Margin of forests 40} & Triploid \\
\hline & $\mathrm{F}-\mathrm{I}$ & Incheon, Kanghwa-gun & Kanghwa-do, Naega, Guhari, Beach & 20 & Diploid \\
\hline & $\mathrm{F}-\mathbb{I}$ & Incheon, Kanghwa-gun & Kanghwa-do, Naega, Guhari, Roadside & 30 & Triploid \\
\hline & G & Incheon, Kanghwa-gun & Kanghwa-do, Yangsa, Inhwasung, Beach & 30 & Triploid \\
\hline & $\mathrm{H}$ & Incheon, Kanghwa-gun & Kanghwa-do, Yangsa, Inhwai, Garden & 20 & Diploid \\
\hline & I & Incheon, Kanghwa-gun & Kanghwa-do, Yangsa, Kyosanri, Margin of forests & 30 & Triploid \\
\hline & J & Incheon, Kanghwa-gun & Kanghwa-do, Songhae, Dangsanri, Sea cliff & 20 & Diploid \\
\hline & $\mathrm{K}-\mathrm{I}$ & Incheon, Kanghwa-gun & Kanghwa-do, Songhae, Hadori, Garden & 20 & Triploid \\
\hline & $\mathrm{K}-$ II & Incheon, Kanghwa-gun & Kanghwa-do, Songhae, Hadori, Garden & 20 & Triploid \\
\hline & A & Incheon, Ongjin-gun & Jangbong-do, Yongam beach, Sea cliff & 30 & Diploid \\
\hline & $\mathrm{B}$ & Incheon, Ongjin-gun & Jangbong-do, Yongam beach, Sea cliff & 30 & Diploid \\
\hline & $\mathrm{C}$ & Incheon, Ongjin-gun & Jangbong-do, Yongam beach, Sea cliff & 30 & Diploid \\
\hline & $\mathrm{D}$ & Incheon, Ongjin-gun & Jangbong-do, Yongam beach, Sea cliff & 30 & Diploid \\
\hline & $\mathrm{E}$ & Incheon, Ongjin-gun & Jangbong-do, Jinchon beach, Sea cliff & 30 & Diploid \\
\hline & $\mathrm{F}$ & Incheon, Ongjin-gun & Jangbong-do, Jinchon beach, Sea cliff & 30 & Diploid \\
\hline & $\mathrm{G}$ & Incheon, Ongjin-gun & Jangbong-do, Jinchon beach, Sea cliff & 30 & Diploid \\
\hline & $\mathrm{H}$ & Incheon, Ongjin-gun & Jangbong-do, Jinchon beach, Sea cliff & 30 & Diploid \\
\hline & I & Incheon, Ongjin-gun & Jangbong-do, Chookdong, Sea cliff & 30 & Diploid \\
\hline & $\mathrm{J}$ & Incheon, Ongjin-gun & Book-do, Sea cliff & 30 & Diploid \\
\hline \multirow[t]{7}{*}{7} & A & Incheon, Ongjin-gun & Yungjong-do, Yedanpo, Sea cliff & 30 & Diploid \\
\hline & $\mathrm{B}$ & Incheon, Ongjin-gun & Yungjong-do, Yedanpo, Sea cliff & 30 & Diploid \\
\hline & $\mathrm{C}$ & Incheon, Ongjin-gun & Yungjong-do, Yedanpo, Sea cliff & 30 & Diploid \\
\hline & $\mathrm{D}$ & Incheon, Ongjin-gun & Yungjong-do, Yedanpo, Sea cliff & 30 & Diploid \\
\hline & $\mathrm{E}$ & Incheon, Ongjin-gun & Yungjong-do, Yedanpo, Sea cliff & 100 & Diploid \\
\hline & $\mathrm{F}$ & Incheon, Ongjin-gun & Yungjong-do, Yedanpo, Sea cliff & 30 & Diploid \\
\hline & $\mathrm{G}$ & Incheon, Ongjin-gun & Yungjong-do, Yedanpo, Sea cliff & 30 & Diploid \\
\hline \multirow[t]{4}{*}{8} & A & Incheon, Ongjin-gun & Eulwang-do, Eulwangri, Sea cliff & 30 & Diploid \\
\hline & $\mathrm{B}$ & Incheon, Ongjin-gun & Eulwang-do, Eulwangri, Sea cliff & 30 & Diploid \\
\hline & $\mathrm{C}$ & Incheon, Ongjin-gun & Eulwang-do, Eulwangri, Sea cliff & 30 & Diploid \\
\hline & $\mathrm{D}$ & Incheon, Ongjin-gun & Eulwang-do, Eulwangri, Sea cliff & 30 & Diploid \\
\hline \multirow[t]{6}{*}{9} & A & Incheon, Ongjin-gun & Mooeui-do, Port, Sea cliff & 30 & Diploid \\
\hline & $\mathrm{B}$ & Incheon, Ongjin-gun & Mooeui-do, Hanakke beach, Beach & 30 & Diploid \\
\hline & $\mathrm{C}$ & Incheon, Ongjin-gun & Mooeui-do, Hanakke beach, Beach & 30 & Diploid \\
\hline & $\mathrm{D}$ & Incheon, Ongjin-gun & Mooeui-do, Hanakke beach, Beach & 30 & Diploid \\
\hline & $\mathrm{E}$ & Incheon, Ongjin-gun & Mooeui-do, Goorackkkuji, Sea cliff & 30 & Diploid \\
\hline & $\mathrm{F}$ & Incheon, Ongjin-gun & Mooeui-do, Goorackkkuji, Sea cliff & 30 & Diploid \\
\hline \multirow[t]{13}{*}{10} & G & Incheon, Ongjin-gun & Deokjuck-do, Mocksum, Beach & 50 & Diploid \\
\hline & $\mathrm{H}$ & Incheon, Ongjin-gun & Deokjuck-do, Mocksum, Sea cliff & 100 & Diploid \\
\hline & I & Incheon, Ongjin-gun & Deokjuck-do, Mocksum, Beach & 30 & Diploid \\
\hline & $J$ & Incheon, Ongjin-gun & Deokjuck-do, Parackkeumi, Beach & 50 & Diploid \\
\hline & $\mathrm{K}$ & Incheon, Ongjin-gun & Deokjuck-do, Parackkeumi, Beach & 50 & Diploid \\
\hline & $\mathrm{L}$ & Incheon, Ongjin-gun & Deokjuck-do, Sundolbawui, Sea cliff & 100 & Diploid \\
\hline & M & Incheon, Ongjin-gun & Deokjuck-do, Sundolbawui, Beach & 50 & Diploid \\
\hline & $\mathrm{N}$ & Incheon, Ongjin-gun & Deokjuck-do, Neungdong, Beach & 50 & Diploid \\
\hline & 0 & Incheon, Ongjin-gun & Deokjuck-do, Seopori, Roadside & 10 & Diploid \\
\hline & $\mathrm{P}$ & Incheon, Ongjin-gun & Deokjuck-do, Seopori beach, Sea cliff & 20 & Diploid \\
\hline & Q & Incheon, Ongjin-gun & Deokjuck-do, Seopori beach, Sea cliff & 20 & Diploid \\
\hline & $\mathrm{R}$ & Incheon, Ongjin-gun & Deokjuck-do, Keunima, Sea cliff & 20 & Diploid \\
\hline & $\mathrm{S}$ & Incheon, Ongjin-gun & Deokjuck-do, Batjireum beach, Sea cliff & 20 & Diploid \\
\hline
\end{tabular}


Table 1. Continued.

\begin{tabular}{|c|c|c|c|c|c|}
\hline $\begin{array}{l}\text { Island } \\
\text { No. }^{z}\end{array}$ & Population & $\begin{array}{l}\text { County, } \\
\text { province }\end{array}$ & $\begin{array}{l}\text { Island, locality } \\
\text { and habitat }\end{array}$ & $\begin{array}{c}\text { Population } \\
\text { size }\end{array}$ & $\begin{array}{l}\text { Ploidy } \\
\text { form }\end{array}$ \\
\hline \multirow[t]{11}{*}{11} & A & Incheon, Ongjin-gun & Jawol-do, Yokol, Sea cliff & 30 & Diploid \\
\hline & B & Incheon, Ongjin-gun & Jawol-do, Yokol, Sea cliff & 30 & Diploid \\
\hline & $\mathrm{C}$ & Incheon, Ongjin-gun & Jawol-do, Yokol, Sea cliff & 30 & Diploid \\
\hline & D & Incheon, Ongjin-gun & Jawol-do, Eoryukol, Sea cliff & 30 & Diploid \\
\hline & $\mathrm{E}$ & Incheon, Ongjin-gun & Jawol-do, Tuckbawui, Sea cliff & 30 & Diploid \\
\hline & $F$ & Incheon, Ongjin-gun & Jawol-do, Hanapo, Beach & 30 & Diploid \\
\hline & $\mathrm{G}$ & Incheon, Ongjin-gun & Jawol-do, Jangkol beach, Beach & 30 & Diploid \\
\hline & $\mathrm{H}$ & Incheon, Ongjin-gun & Jawol-do, Sogonhwon, Beach & 30 & Diploid \\
\hline & I & Incheon, Ongjin-gun & Jawol-do, Sogongwon, Sea cliff & 30 & Diploid \\
\hline & $\mathrm{J}$ & Incheon, Ongjin-gun & Jawol-do, Dalbawui, Sea cliff & 30 & Diploid \\
\hline & $\mathrm{K}$ & Incheon, Ongjin-gun & Jawol-do, Mocksum, Sea cliff & 30 & Diploid \\
\hline \multirow[t]{4}{*}{12} & $\mathrm{~A}$ & Incheon, Ongjin-gun & Youngheung-do, Naedong, Beach & 20 & Diploid \\
\hline & $\mathrm{B}$ & Incheon, Ongjin-gun & Youngheung-do; Naedong, Beach & 50 & Diploid \\
\hline & $\mathrm{C}$ & Incheon, Ongjin-gun & Youngheung-do, Sibripo beach, Beach & 50 & Diploid \\
\hline & $\mathrm{D}$ & Incheon, Ongjin-gun & Youngheung-do, Sibripo beach, Beach & 50 & Diploid \\
\hline \multirow[t]{13}{*}{13} & A & Incheon, Ongjin-gun & Backa-do, Earyu, Sea cliff & 10 & Diploid \\
\hline & $\mathrm{B}$ & Incheon, Ongjin-gun & Backa-do, Earyu, Sea cliff & 20 & Diploid \\
\hline & $\mathrm{C}$ & Incheon, Ongjin-gun & Backa-do, Gaegunneor, Sea cliff & 20 & Diploid \\
\hline & D & Incheon, Ongjin-gun & Backa-do, Gaegunneor, Sea cliff & '20 & Diploid \\
\hline & $\mathrm{E}$ & Incheon, Ongjin-gun & Backa-do, Gaegunneor, Sea cliff & 20 & Diploid \\
\hline & $\mathrm{F}$ & Incheon, Ongjin-gun & Backa-do, Gaegunneor, Sea cliff & 20 & Diploid \\
\hline & G & Incheon, Ongjin-gun & Backa-do, Gaegunneor, Sea cliff & 20 & Diploid \\
\hline & $\mathrm{H}$ & Incheon, Ongjin-gun & Backa-do, Gaegunneor, Sea cliff & 20 & Diploid \\
\hline & I & Incheon, Ongjin-gun & Backa-do, Mockgasi, Sea cliff & 20 & Diploid \\
\hline & $\mathrm{J}$ & Incheon, Ongjin-gun & Backa-do, Mockgasi port, Beach & 20 & Diploid \\
\hline & $\mathrm{K}$ & Incheon, Ongjin-gun & Backa-do, Mockgasi, Sea cliff & 20 & Diploid \\
\hline & $\mathrm{L}$ & Incheon, Ongjin-gun & Backa-do, Keunmal, Sea cliff & 20 & Diploid \\
\hline & M & Incheon, Ongjin-gun & Backa-do, Keunmal, Sea cliff & 20 & Diploid \\
\hline \multirow[t]{3}{*}{14} & A & Choongnam, Dangjin-gun & Daenanji-do, port, Sea cliff & 10 & Diploid \\
\hline & B & Choongnam, Dangjin-gun & Daenanji-do, port, Sea cliff & 10 & Diploid \\
\hline & $\mathrm{C}$ & Choongnam, Dangjin-gun & Daenanji-do, port, Sea cliff & 10 & Diploid \\
\hline \multirow[t]{10}{*}{$15^{x}$} & A & Choongnam, Taeahn-gun & Iwon, Naeri, Sea cliff & 300 & Diploid \\
\hline & B & Choongnam, Taeahn-gun & Iwon, Baemyunri, Sea cliff & 500 & Diploid \\
\hline & $\mathrm{C}$ & Choongnam, Taeahn-gun & Wonbook, Hwangchonri, Beach & 200 & Diploid \\
\hline & $\mathrm{D}$ & Choongnam, Taeahn-gun & Wonbook, Hakampo, Beach & 200 & Diploid \\
\hline & $\mathrm{E}$ & Choongnam, Taeahn-gun & Wonbook, Sinduri, Beach & 50 & Diploid \\
\hline & $\mathrm{F}$ & Choongnam, Taeahn-gun & Sowon, Euihangri, Sea cliff & 30 & Diploid \\
\hline & G & Choongnam, Taeahn-gun & Sowon, Backripo, Sea cliff & 30 & Diploid \\
\hline & $\mathrm{H}$ & Choongnam, Taeahn-gun & Nammyun, Magumpo, Sea cliff & 1,000 & Diploid \\
\hline & $\mathrm{I}-\mathrm{I}$ & Choongnam, Taeahn-gun & Nammyun, Gomsum, Sea cliff & 500 & Diploid \\
\hline & $\mathrm{I}-\mathrm{II}$ & Choongnam, Taeahn-gun & Nammyun, Gomsum, Sea cliff & 2,000 & Diploid \\
\hline \multirow[t]{3}{*}{16} & A & Choongnam, Taeahn-gun & Ahnmyun-do, Hwangpo, Sea cliff & 1,000 & Diploid \\
\hline & B & Choongnam, Taeahn-gun & Ahnmyun-do, Sinyari, Sea cliff & 200 & Diploid \\
\hline & $\mathrm{C}$ & Choongnam, Taeahn-gun & Ahnmyun-do, Geatga, Beach & 1,000 & Diploid \\
\hline
\end{tabular}

$z$ Island No. corresponds to the number in Fig. 1.

${ }^{y}$ Population size estimated by approximate number of reproductive individuals.

x Population A to $\mathrm{G}$ in island 15 were located in seaside of Taeahn peninsula, and population $\mathrm{H}$, I-I and I-II were located in small islands. 
Table 2. Classification and observation frequency of habitat types of L. lancifolium populations observed in 16 islands of the Bay of Kyunggi.

\begin{tabular}{crc}
\hline Type of natural habitat & No. of population & \% of population \\
\hline Sea cliff & 103 & 72.5 \\
Beach & 28 & 19.7 \\
Roadside & 3 & 2.1 \\
Forest & 2 & 1.4 \\
Others $^{2}$ & 6 & 4.2 \\
\hline Total & 142 & 100 \\
\hline
\end{tabular}

z Habitats such as grassy slopes, gardens and riverside.

environments lead us to suppose that insular populations of $L$. lancifolium seem to have been established from seeds and bulbils dispersed by the forces of sea waves and currents running north to south along the west coasts of the Korean Peninsula.

Sea cliff populations were growing in the narrow zones from $2-3 \mathrm{~m}$ above sea level to the margin of the forests within $10 \mathrm{~m}$ above sea level. Such sites presumably give high salinity stress. In those habitats, thus, maritime species such as Gypsophila oldhamiana, Dianthus japonicus, Aster spathulifolius, Crepidiastrum lancedatum, Sedum kamtschaticum, Allium monanthum and Chrysanthemum boreale often grow together with $L$. lancifolium. These herbal plants live toughly in the little sediment in the crack of large rocks. In several beach populations of Ahnmyun-do (Isl. No. 16), the larger bulbs were submerged more than one meter below the ground. The fact suggests the beach populations have been autonomously established and preserved for a long time.

\section{Geographic distribution of diploid L. lancifolium}

One hundred and sixteen (82\%) of 142 populations consisted of diploid forms, and triploid forms were restricted only in three islands (Table 1, Fig. 1). In the northernmost islands of South Korea, Backryung-do (Isl. No. 1) and Sochung-do (Isl. No. 2), there were no diploid, but only triploid individuals. Eight diploid and six triploid populations occupied in Kanghwa-do (Isl. No. 5), the nearest island to the mainland of South Korea. Triploid plants in these islands set no capsules and were growing in the habitats similar to those in the other islands inhabited by diploid forms; i.e., most were growing in sea cliffs.

There has been no report on the geographic distribution of diploid L. lancifolium except that by Noda (1986). Thus, this study gives additional fact on the geographic distribution of diploid L. lancifolium.

Only triploid form has been found in the inland of South Korea (Noda, 1986; Kim et al., 2004). It seems that Kanghwa-do (Isl. No. 5) and the neighbor islands (Isl. No. 3, 4) are the approximate northern limit of the distribution of diploid L. lancifolium and the middle western to southern coasts and islands of the Korean Peninsula may be the origin of diploid L. lancifolium. To make clear this point, the further investigation for the coastal regions of Yellow Sea, especially in Sandong Peninsula in China and the western islands and coasts in North Korea is necessary. 


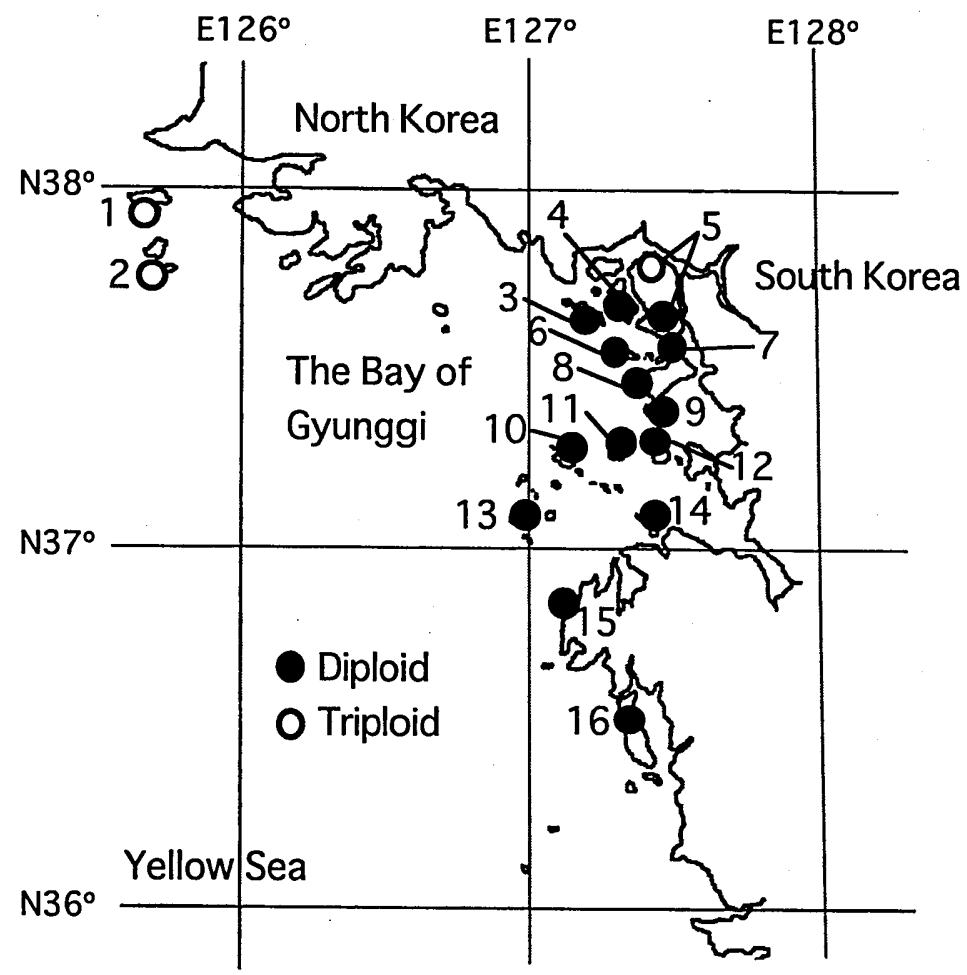

Fig. 1. Geographic distribution of diploid and triploid L. lancifolium in 16 islands of the Bay of Kyunggi.

\section{REFERENCES}

Kim, J. H., H. Y. Kyung, Y. Xuan, Y. Choi, Y. C. Park, M. Hiramastu, and K. O. Yoo 2004 Studies on the distribution and dispersal of Korean Lilium lancifolium Thunb. J. Kor. Flower Res. Soc., 12(2): 127-141 (in Korean with English abstract)

Lightly, R. W. 1968 The lilies of Korea. Lily Year Book Roy. Hort. Soc., 31: 31-39

Noda, S. 1978 Chromosomes of diploid and triploid forms found in the natural populations of tiger lily in Tsushima. Bot. Mag. Tokyo, 91: 279-283

Noda, S. 1986 Cytogenetic behavior, chromosome differentiations, and geographic distribution in Lilium lancifolium (Liliaceae). Plant Species Biology, 1: 69-78

Noda, S. 1991 Chromosomal variation and evolution in the genus Lilium. In "Chromosome Engineering in Plants: Genetics, Breeding, Evolution. Part B", ed. by T. Tsuchiya and P. K. Gupta, Elsevier, Amsterdam, pp. 507-524

Noda, S. and H. S. Lee 1980 Relationship between cytogenetic structure of three species in Liliaceae and human activities. In "Reports for a Grant-in-Aid for Scientific Research from the Ministry of Education, Science, Sports and Culture of Japan, History on the successive changes of environments in Korea", pp. 33-55 (in Japanese)

Ozaki, Y., K. Narikiyo, M. Hiramatsu, K. Ureshino and H. Okubo 1998 Application of flow cytometry for rapid determination of ploidy levels in asparagus (Asparagus officinalis L.). J. Fac. Agr., Kyushu Univ., 43: 83-88 
Song, N. H. 1997 Chromosomal differentiation and geographic distribution of Lilium lancifolium Thunberg native to Cheju island in Korea. Res. Sci. Math. Educ., Taegu Nat. Univ. Educ., 20: 93-105

Wilson, E. H. 1925 The lilies of Eastern Asia. A monograph. Dulau \& Comp., London, UK pp. 110 